
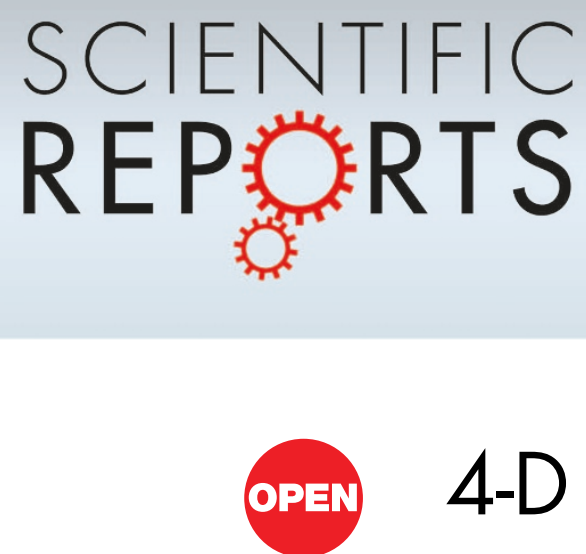

\title{
4-D Photoacoustic Tomography
}

\author{
Liangzhong Xiang, Bo Wang, Lijun Ji \& Huabei Jiang
}

SUBJECT AREAS:

IMAGING AND SENSING

PRE-CLINICAL STUDIES

APPLIED PHYSICS

BIOMEDICAL ENGINEERING

Received

15 November 2012

Accepted

17 December 2012

Published

23 January 2013

Correspondence and requests for materials should be addressed to H.J. (hiiang@bme.ufl.

edu)
J. Crayton Pruitt Family Department of Biomedical Engineering University of Florida Gainesville, FL 3261 1, U.S.A.

Photoacoustic tomography (PAT) offers three-dimensional (3D) structural and functional imaging of living biological tissue with label-free, optical absorption contrast. These attributes lend PAT imaging to a wide variety of applications in clinical medicine and preclinical research. Despite advances in live animal imaging with PAT, there is still a need for 3D imaging at centimeter depths in real-time. We report the development of four dimensional (4D) PAT, which integrates time resolutions with 3D spatial resolution, obtained using spherical arrays of ultrasonic detectors. The 4D PAT technique generates motion pictures of imaged tissue, enabling real time tracking of dynamic physiological and pathological processes at hundred micrometer-millisecond resolutions. The 4D PAT technique is used here to image needle-based drug delivery and pharmacokinetics. We also use this technique to monitor 1) fast hemodynamic changes during inter-ictal epileptic seizures and 2) temperature variations during tumor thermal therapy.

AT is a 3D imaging technique based on the photoacoustic effect, a phenomena first described by Alexander Graham Bell over one hundred years ago ${ }^{1-3}$. Within the last decade, the photoacoustic effect has been successfully used for in vivo biomedical imaging of different biological tissues ${ }^{4-6}$. Photoacoustic imaging methods are characterized as: 1) photoacoustic microscopy (or PAM), used to image at millimeter imaging depths at micrometer resolution ${ }^{7,8}$ and 2) photoacoustic tomography (PAT), used at centimeter imaging depths with applications for breast tumor detection ${ }^{9}$, peripheral joint imaging ${ }^{10,11}$ and whole-body mouse imaging ${ }^{12,13}$. Early PAT systems used single ultrasonic transducers, and required mechanical scanning and long data acquisition times were necessary to obtain high-quality images ${ }^{5,14}$. Recently, array-based PAT systems have been developed to reduce the imaging time. In addition, high frame-rate photoacoustic (PA) imaging has been performed in $2 \mathrm{D}$ using linear arrays ${ }^{15,16}$. Despite advances in live animal imaging with PAT, there is still a need for 3D imaging at centimeter depths in real-time.

Metal needles are commonly used for localized drug delivery in cancer therapy, and for tissue collection in biopsies ${ }^{17}$. Accurate and real-time information on needle position and orientation is important for proper needle guidance and placement within tissue. Recently, photoacoustic imaging has been used to visualize metal needles in vivo ${ }^{18,19}$. Most current photoacoustic systems provide 2D cross-sectional images of tissue. However, 3D images would afford better monitoring during needle insertion and injection ${ }^{20,21}$. Therefore, 3D PAT represents an important advance in needle guidance systems.

PAT has also been successfully used for non-invasive brain imaging in animal models of epilepsy ${ }^{22}$. Hemodynamic parameters associated with neuronal metabolism are often used to study epileptic seizures. During siezures, epileptiform events occur quickly and existing PAT imaging systems are not able to monitor these rapid changes in neuronal hemodynamics ${ }^{22}$. Real-time imaging methods that can resolve neural activities in three dimensions would be advantageous for the study of epilepsy, especially for the detection of spontaneous epileptic seizures.

Studies have also demonstrated the use of PAT in image-guided photothermal therapies in both animals and excised tissue ${ }^{23-25}$. In previously PAT systems, 2D thermal maps are generated by monitoring temperatureinduced changes in photoacoustic signals during thermal therapy procedures ${ }^{26,27}$. However, $2 \mathrm{D}$ visualization of the heating pattern cannot effectively steer of heating focus into the tumor. This often left parts of the tumor unheated while generating too much heat in the surrounding normal tissues. Thus, 3D temperature distributions are desired to provide more comprehensive temperature monitoring during clinical applications.

Real-time 3D photoacoustic imaging has the potential to capture the physiology and pathology associated with dynamic phenomena in three-dimensional space. However, existing 3D PAT systems have long data collection times (from $24 \mathrm{~s}$ up to $8 \mathrm{~min})^{12}$. To monitor dynamic processes, the dimension of time must be integrated into 3D photoacoustic tomograms ${ }^{28}$. Although simultaneous real-space and real-time resolution can be obtained using sparse arrays of ultrasonic detectors (only 15 detectors), the spatial resolution of these systems is poor (2$3 \mathrm{~mm})^{29}$.

Here we report the development of $4 \mathrm{D}$ PAT, a technique in which, the time resolution is integrated with $3 \mathrm{D}$ spatial resolutions obtained from a series of $2 \mathrm{D}$ projections. The methodology is demonstrated in: 1 ) $3 \mathrm{D}$ needle 
guiding during drug delivery; 2) monitoring dynamic events during epileptic seizures; and 3) monitoring dynamic tissue temperature changes during tumor thermal therapy.

\section{Results}

Concept of 4D PAT. Imaging System and its characterization. In the 4D PAT imaging system, excitation light originates from a tunable (680-900 nm) Ti: Sapphire laser (LOTIS TII Ltd, Minsk, Belarus), delivering $<6.5 \mathrm{~ns}$ duration pulses with repetition frequency of $10 \mathrm{~Hz}$. The beam is guided into a custom-made silica fused-end fiber bundle to create a cylindrical-shaped illumination pattern of approximately $20 \mathrm{~mm}$ in diameter upon the surface of the imaged object (Fig. 1). To achieve calibration for PAT imaging reconstruction, the energy of each laser pulse is detected by a photodiode. A 192 element 3D sphere transducer array is used to capture the PA signals generated by the laser light. The 192 transducers placed along a custom-fabricated white ABS spherical interface containing 610 through holes with counter bores, formed 11 evenly spaced layers along the vertical direction of the ball as shown in Supplemental Fig. 1(a), online. The transducer positions on the spherical interface are selectable which depends on geometry of the imaged target. The transducers were glued onto the interface with epoxy which can be removed to allow the position change of the transducers. The interface has an outer diameter of $160 \mathrm{~mm}$ and an inner diameter of $140 \mathrm{~mm}$. The physical width of the light beam measured $\sim 20 \mathrm{~mm}$ at the center-of-curvature of the sphericalarray. In this way, an imaging region of about $\pi \times 10 \times 10 \times$ $30 \mathrm{~mm}^{3}$ can be covered using a single laser pulse. A 192 channel data acquisition system consists of preamplifiers, secondary stage amplifiers (for optimizing the signal-to-noise ratio), and a $3: 1$ electronic multiplexer coupled with a 64-channel analog-to-digital converter. The average speed of PAT data acquisition system is $0.33 \mathrm{~s} /$ frame and was limited by the $10 \mathrm{~Hz}$ laser repetition ${ }^{30}$. The spatial resolution of the PAT system is inversely proportional to the bandwidth of the ultrasonic transducer. Each ultrasonic detector has a $5 \mathrm{MHz}$ central frequency and a $70 \%$ nominal bandwidth with an active area of $3 \mathrm{~mm}$ in diameter and total diameter of about $6 \mathrm{~mm}$ (Blatek, Inc., PA, U.S.A.). As a result, the spatial resolution of this system is better (smaller) than $0.19 \mathrm{~mm}$ in the $\mathrm{x}-\mathrm{y}$ direction, and better (smaller) than $0.27 \mathrm{~mm}$ in $\mathrm{z}$ direction (see supplemental data Figure 1, online). For in vivo experiments, the rat head was elevated to the center of the spherical interface through a chamber fixed at the tank bottom, whose top was about $15 \mathrm{~mm}$ beneath the interface center, and a transparent plastic wrap was used to cover the chamber top. Ultrasound gel was used to couple the sound from the animal to the plastic wrap. The ultrasonic transducer array with the spherical interface, placed in the water bath, was therefore acoustically coupled to the mouse tissues.

3-D Spatial phase-controlled algorithm. The photoacoustic effect is intrinsically three-dimensional and is a product of the optical

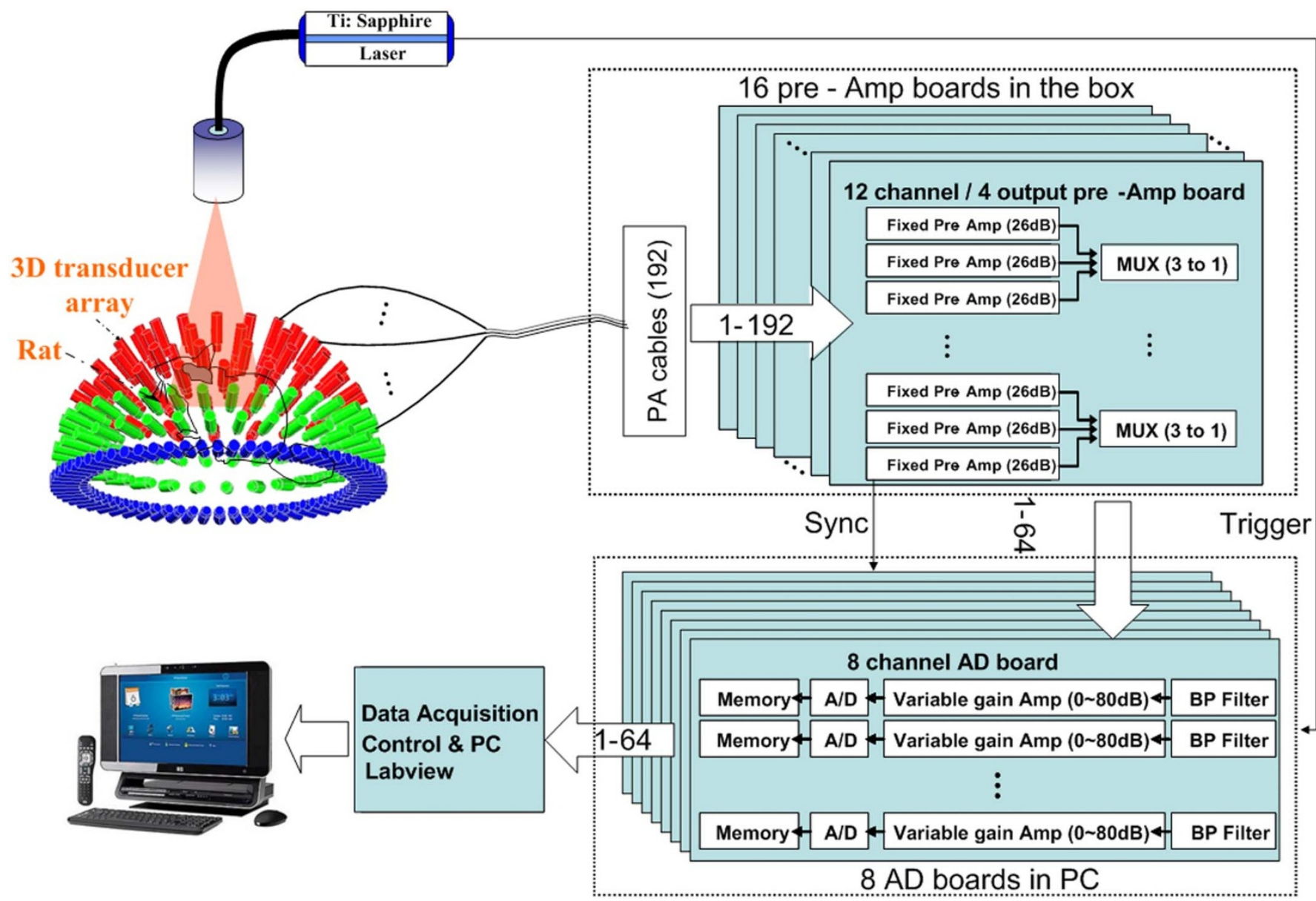

Figure 1 4D PAT system. (a) Schematic of the 4D PAT system. Pulsed laser light from a Ti: Sapphire laser tunable (690 to $950 \mathrm{~nm})$ is delivered to tissue surfaces through an optical fiber. A 192 element 3D sphere transducer array is used to capture the PA signals generated by the laser light. A 192 channel data acquisition system consists of preamplifiers, secondary stage amplifiers (for optimizing the signal-to-noise ratio), and a $3: 1$ electronic multiplexer coupled with a 64-channel analog-to-digital converter. The average speed of PAT data acquisition system is $0.33 \mathrm{~s} /$ frame and was limited by the $10 \mathrm{~Hz}$ laser repetition. The spatial resolution of this system is better (smaller) than $0.19 \mathrm{~mm}$ in the $\mathrm{x}-\mathrm{y}$ direction, and better (smaller) than $0.27 \mathrm{~mm}$ in $\mathrm{z}$ direction (Supplemental data Figure 1, online). 
radiation scattered by the tissue being imaged. Photoacoustic waves are spherical in nature and propagate in all directions from their point of origin. In 3-D spatial phase-controlled algorithm, any detector should have recorded all light-absorbing objects in the detection area of the transducer according to spherical PA source theory. Therefore, different optical absorbers in the same cross section can be simultaneously gathered by the corresponding element, and the optical absorbers at various spatial locations can be resolved by measuring the time of arrival of PA pulses at each activated detector of the transducer assuming constant ultrasonic velocity in tissue. The reconstruction image pixel, $P_{m}$ is calculated through the following formula ${ }^{31}$ :

$$
P_{m}=\sum_{k=1}^{K} D(\theta)_{k} P\left(t_{m k}\right)
$$

where $m$ is the spatial vector of the PA source, $k$ is the position of the $k$ th detector, and $K$ is the total number of working detectors. $D(\theta)$ is defined as the projection intensity weight function of $\theta$, the projection angle which is no more than the maximal acceptance angle of the array element. $P\left(t_{m k}\right)$ is the signal value collected by the $k t h$ detector at position $m . t_{m k}=r_{m k} / v$ represents the time when PA pulses spread from position $m$ to $k . r_{m k}$ is the distance between PA source at position $m$ and the $k$ th detector. $v$ is the average velocity of the acoustic wave in tissue. According to Eq. (1), the volumetric data collected by the spherical transducer array in a single laser pulse can be used to reconstruct $2 \mathrm{D}$ projection images at various $Z$ axis depths. The concept of the 4D PAT is illustrated in Fig. 2, which depicts the construction of tomograms from $2 \mathrm{D}$ projections at different angles and times. Because of the various dimensions involved, we note that at a given time each $2 \mathrm{D}$ projection represents a $3 \mathrm{D}$ frame (including time), whereas a $3 \mathrm{D}$ tomogram when constructed from all the $2 \mathrm{D}$ projections represents a $4 \mathrm{D}$ frame.

As shown in Fig. 2, a series of PA images of various 2D projections is generated at different angles along $z$ axis, allowing the construction of $3 \mathrm{D}$ tomograms over a series of times, $t_{i}^{\theta}$, where $i$ denotes a given time and $\theta$ denotes the tilt angle with respect to the $Z$ axis.

Calibration was performed using a point object located at the center of the spherical array, which ensured an isotropic acoustic emission profile for all directions. We measured and compensated

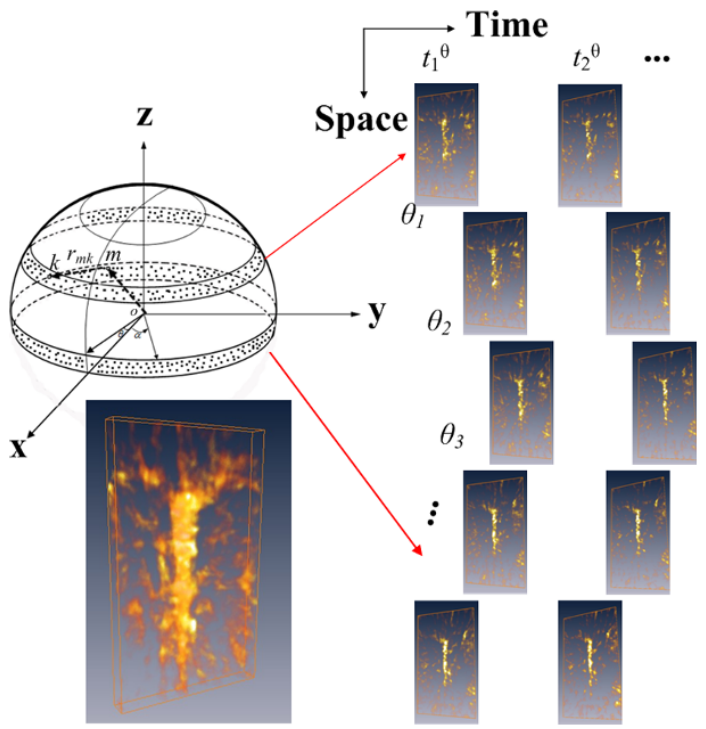

Figure $2 \mid$ Schematic representation of time-resolved 4D photoacoustic tomography. 3-D spatial phase-controlled algorithm was used to reconstruct the imaging. A series of $2 \mathrm{D}$ images at various projection angles, $\theta$ and time steps $t$ can be obtained to construct the 4D movies of objects in motion. $r_{m k}$ is the distance between photoacoustic source at position $m$ and No. $k$ detector.
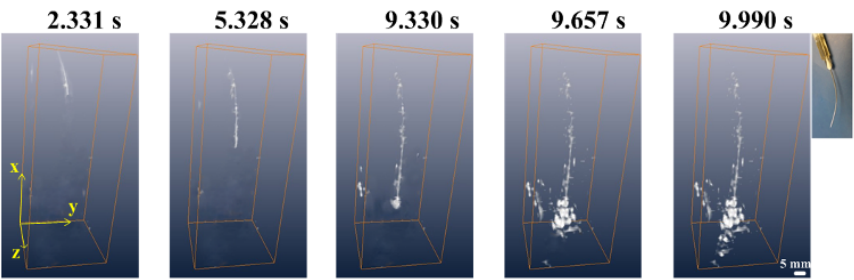

Figure $3 \mid$ Image-guiding curved needle insertion and drug delivery. 3D PA guidance of a curved needle, a snapshot of needle insertion is tracked in real-time (Video 1). From left to right, the needle positions were monitored at different time serials from $2.331 \mathrm{~s}$ to $9.990 \mathrm{~s}$. scale bars throughout figure, $2 \mathrm{~mm}$.

the delay of time for all the 192 channels in the radial direction. Using the spatial phase-controlled algorithm, the 3D (volume) tomograms were reconstructed from the aligned/calibrated images. We then used the time series $3 \mathrm{D}$ tomograms to make motion pictures that reflect the temporal evolution of dynamic processes.

Experimental results. Drug delivery monitoring. Real-time 3D images of photoacoustic-guided curved needle insertion are shown in Fig. 3. Video 1 shows a $27 \mathrm{G}$ needle inserted into phantom tissue and tracked photoacoustically in real time. The phantom was made with a lipid emulsion solution (Intralipid) to mimic tissue scattering (optical scattering coefficient $\mu_{s}^{\prime}=1.0 \mathrm{~mm}^{-1}$ ), India ink (optical absorption coefficient $\mu_{a}=0.007 \mathrm{~mm}^{-1}$ ) to simulate tissue absorption and Agar powder (2\%) to solidify the mixed Intralipid and India ink $^{32}$. There is excellent contrast $11.5 \pm 5$ (standard deviation) in the PA response between the needle and the background tissue. The curved needle progression and the drug (ICG) delivery information is detected photoacoustically during the experiment. ICG pharmacokinetics was also monitored within a rat brain in real time with $810 \mathrm{~nm}$ (Fig. 4, Video 2). During the first 20 seconds the signal is constant while a sudden increase of the signal strength occurs right afterward due to the ICG injection, followed by a plateau in the photoacoustic signal strength. Also presented are two time frames of the 3D structures obtained from representative, reconstructed $4 \mathrm{D}$ tomograms at $t=19.647 \mathrm{~s}$ (Fig. 4a) and $t=30.303 \mathrm{~s}$ (Fig. 4b). Immediately after injection, ICG accumulation in the brain cortex is observed photoacoustically. The PA image of the ICG-dyed brain acquired at 30.303 s postinjection shows ICG accumulation enhanced the PA signal (area B) by $8.5 \pm 2.6$ (standard deviation). Video 3 shows a three dimensional rendering of the ICG accumulation at a given time point. After transient accumulation of ICG, the amount of drug reduces exponentially. 2D PAT images extracted from $3 \mathrm{D}$ rat cortical vasculature tomograms (Fig. 4c) matched well with anatomical photographs (Fig. 4d). The brain structures, including the middle cerebral artery, right hemispheres, left hemispheres, left olfactory bulbs, and right olfactory bulbs, are clearly shown in the PAT images (Figure 4c). The micro-blood vessels with diameters less than 100im are also evident (marked 1-3), and correspond well with rat brain anatomical photographs (Figure $4 \mathrm{~d}$ ).

Disease diagnosis and therapy monitoring. To monitor epileptic activity, we induced generalized seizures in rats $(n=10)$ using intraperitoneal injection of pentylenetetrazol (PTZ) and monitored the seizures over time by photoacoustic imaging with $755 \mathrm{~nm}$ laser wavelength. Representative time frames of 2D PA projections of neuronal events were taken at 2 second intervals and are displayed in Fig. 5d. Within 2-3 minutes post-injection, small negative deflections $(0.1-0.2 \mathrm{mV})$ in the local field potential (f.p.) were evident and rapidly developed into stable, well-formed biphasic interictal spikes (1-3 mV) occurring at regular intervals $(0.8-1.5 \mathrm{~Hz}$ ) (Fig. 5b, upper). Each interictal spike was followed by a distinct period of low signal, due to the enhanced absorption of active neural tissue 

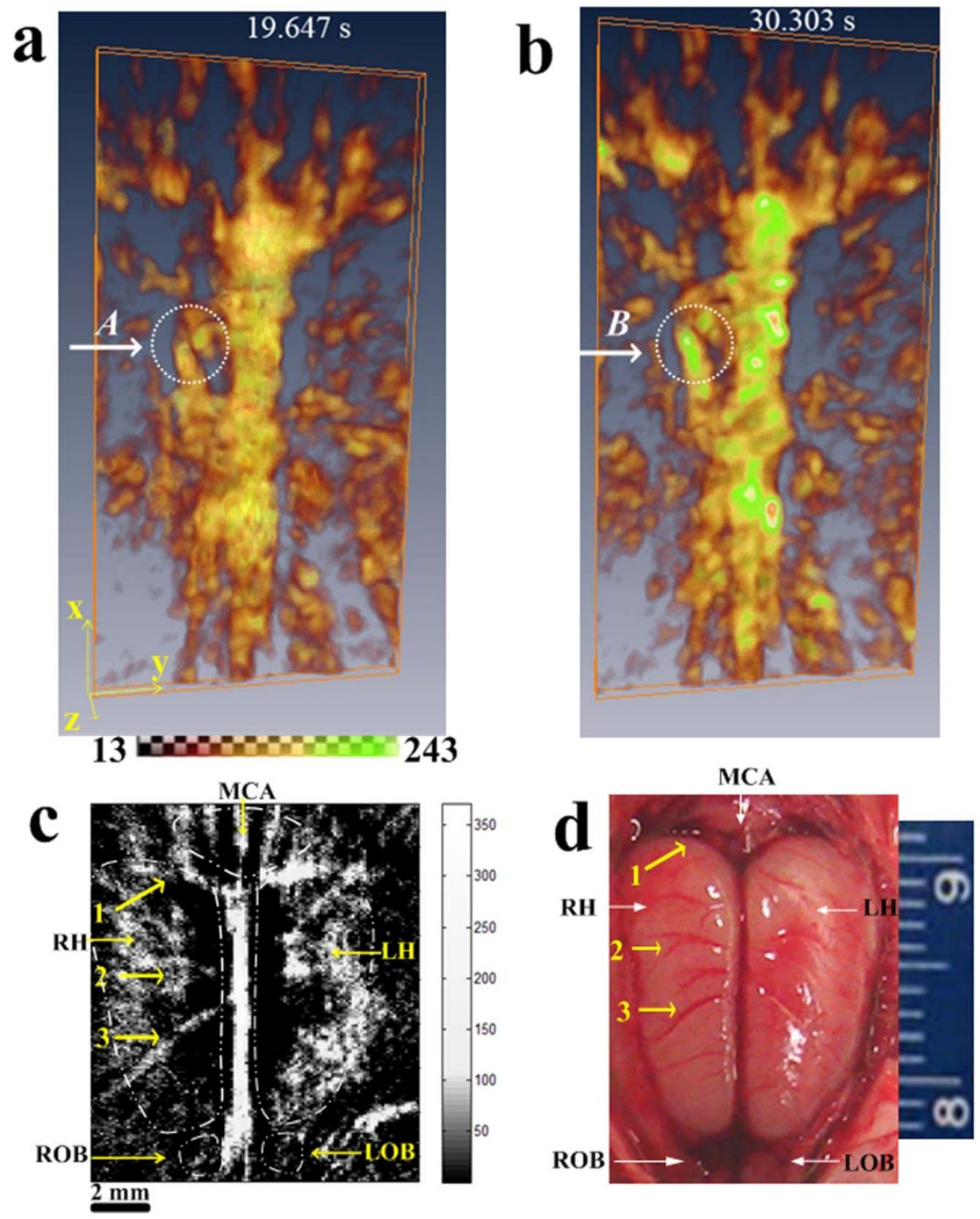

Figure 4 4D visualization of ICG-based pharmacokinetics of drugs. (a) Representative time frames of 3D volume images taken at $t=19.647 \mathrm{~s}$ and (b) $\mathrm{t}=30.303 \mathrm{~s}$ for brain vascular structures. ICG pharmacokinetics was also monitored within a rat brain in real time with $810 \mathrm{~nm}$ (Video 2 ). The PA image of the ICG-dyed brain acquired at 30.303 s post-injection shows ICG accumulation enhanced the photoacoustic signal (area B) by $8.5 \pm 2.6$ (standard deviation). Video 3 shows a three dimensional rendering of the ICG accumulation at a given time point. (c) The 2D noninvasive PAT image extracted from the 3D tomogram of the rat cortical vasculature (d) Open-skull photograph of the rat brain surface acquired after the PAT experiment. Numbers 1-3 indicate the corresponding blood vessels in the PAT image and rat brain photograph. Scale bars in figure 4(c), $2 \mathrm{~mm}$.

(Fig. 5b, lower). As the interictal spikes increased in amplitude and width, so did the intensity of the photoacoustic signal. Thus, there was significant positive correlation between interictal spike amplitude and photoacoustic signal intensity (Fig. 5b). Averaging over multiple interictal spikes, the mean amplitude was determined and the time course of the photoacoustic signal associated with each interictal spike (Fig. 5c) was calculated. Optical absorption changes $(\Delta R / R)$ appeared within 0.5 seconds of the interictal spike and peaked within 1 second of the spike (Fig. 5c). By dividing the single frames occurring after each event by the frame preceding the event, we produced spike-triggered (ST) photoacoustic maps (Fig. 5d). ST maps reveal a clear photoacoustic signal with dynamic changes after each interictal spike. These signals most likely correspond to a population of neurons participating in the paroxysmal event.

$4 \mathrm{D}$ photoacoustic imaging was also used to monitor tissue temperature variations during tumor photothermal therapy on mouse tumor. Fig. $6 \mathrm{a}_{1-5}$ shows representative $2 \mathrm{D}$ slices and Fig. $6 \mathrm{a}_{7}$ is $3 \mathrm{D}$ reconstructed photoacoustic image of mouse mammary tumors at $810 \mathrm{~nm}$ light illumination. Using the optical absorption differences between tumors and surrounding normal tissue, the tumor margins can be easily identified (Fig. $6 \mathrm{a}_{1-6}$ ) and corresponds well with tumor histology (Fig. $6 \mathrm{a}_{8}$ ) and our previous results ${ }^{33}$.

Sliver nanoparticle was used to effectively enhance photothermal therapy because of their high optical absorption in our experiments. Thirty minutes after sliver nanoparticle injection, photothermal therapy was performed for 5 minutes using a CW laser wavelength of $755 \mathrm{~nm}$. During therapy, photoacoustic signals at pulsed laser wavelength of $810 \mathrm{~nm}$ were collected constituently. The CW laser beam spot size on the skin of the mouse was $0.8 \mathrm{~mm}$ in diameter. Based on temperature calculation models ${ }^{25}$, a temperature distribution was estimated. A maximum temperature rise of $6.6^{\circ} \mathrm{C}$ during therapy was observed by photoacoustic-based thermal imaging (Fig. 6c). During the first 1 minute 40 seconds, mouse tumor temperature gradually increased, and then the temperature was relatively constant until the CW laser was turned off.

\section{Discussion}

4D-PAT, which combines the power of volume imaging with time resolution, reveals $3 \mathrm{D}$ physiologic and pathologic phenomena in a living animal. In the drug delivery application demonstrated here, 


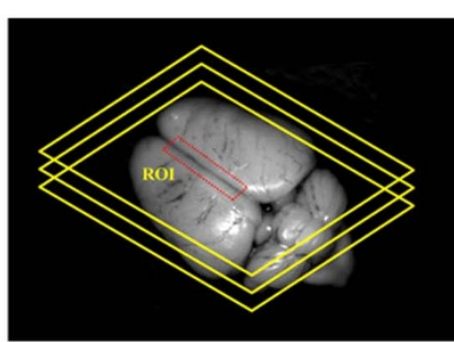

b
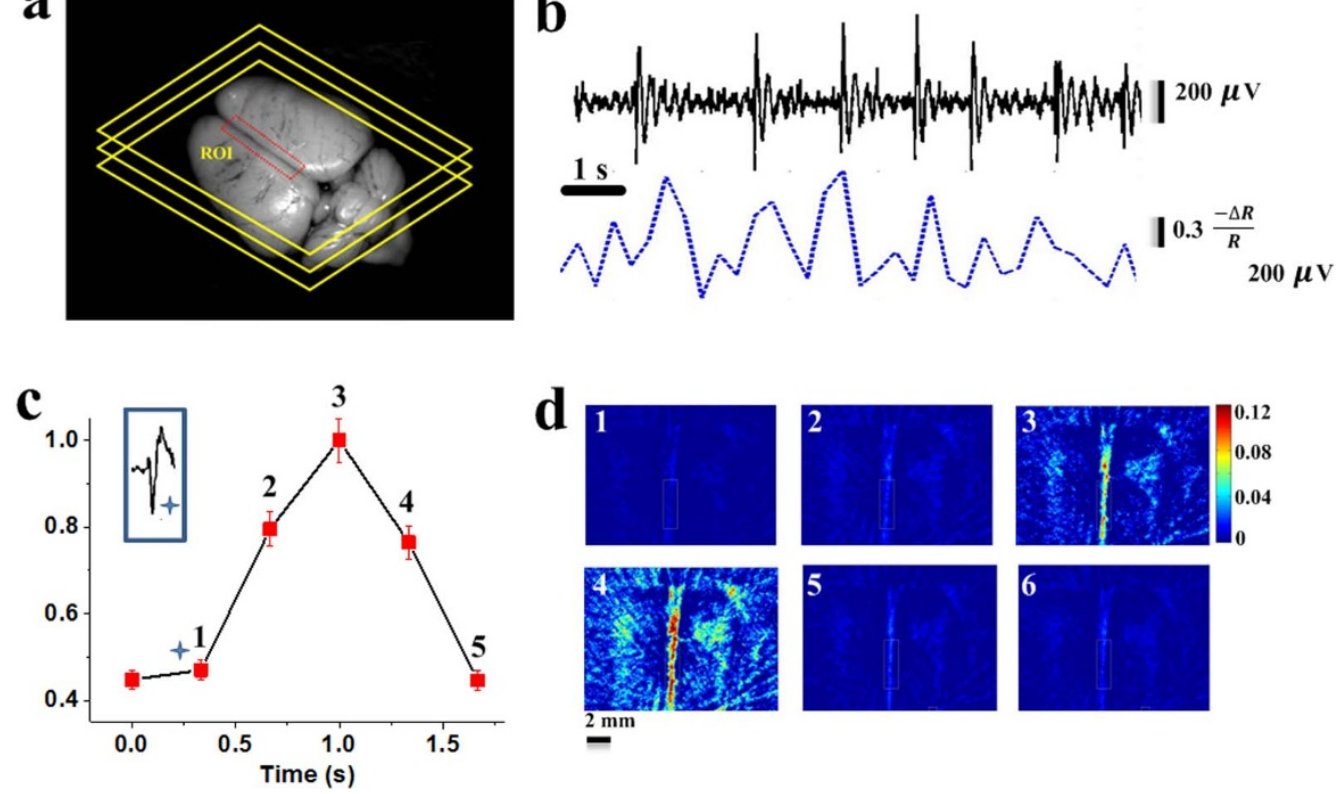

Figure 5 Interictal events monitoring. (a) Schematic showing the location of the imaged region. (b) Simultaneous photoacoustic signal (below) and EEG recording (upper) show that each interictal spike had discrete photoacoustic correlates. (c) Change in reflectance ( $-\Delta \mathrm{R} / \mathrm{R})$ as a function of time for the $0.5 \mathrm{~s}$ before and $1 \mathrm{~s}$ after each spike. Error bars ( \pm s.d.) were calculated from 5 consecutive spikes. Notice the rapid onset of the signal.*, and the time of occurrence of the spike (shown on the left). Numbers correspond to images in next panel. (d) Spike-triggered (ST) epilepsy PA maps were obtained by dividing each frame after the spike by the frame prior to the spike (denominator frame). A significant increase of optical absorption is seen during the interictal onset, scale bars throughout figure, $2 \mathrm{~mm}$.

the tomographic motion picture reveals ICG pharmacokinetics over time. This technique is able to monitor phenomena that change rapidly, such as hemodynamic changes during inter-ictal epilepsy seizures and temperature variations during tumor thermal therapy. In this report, 4D PAT images were generated by integrating time resolution with $3 \mathrm{D}$ spatial resolutions obtained from a complete tilt series of $2 \mathrm{D}$ projections of a living animal. The methodology promises to have wide-ranging applications in fundamental biological research and clinical practice.

Here we show that 4D PAT is able to monitor clinically-relevant curved needle insertion and drug delivery in real time. Indeed, the PA signal from the metal needle is strong due to the high optical absorption of the needle in contrast to the surrounding tissue constituents ${ }^{18}$. The real time 3D PAT imaging system described here overcomes the limits of existing 2D PAT imaging. With this system, the physician can now view curved needle locations within tissues in real time (Fig. 3 and Video 1) offering a powerful tool for identifying metal implants and their location relative to other tissue structures. Combining with the conventional ultrasound imaging, our current PAT system can easily identify metal implants and their surroundings which are valuable in image-guided therapy ${ }^{34,35}$. This technique is highly translatable to clinical applications, e.g. image-guided SLN biopsy and nerve blockings.

We show 3D real-time tracking of ICG-based drug biodistribution and pharmacokinetics using PAT. A clear advantage of this in vivo approach is the opportunity of direct visualization of disease processes on the molecular scale and the quantification of changes to the relevant marker molecules in a non-invasive test environment. In the future, other drugs with optical absorption contrast could be developed for photoacoustic imaging applications. However, additional studies will be required to evaluate the efficiency, BBB penetration capabilities, dosing, safety and toxicity of these new contrast agents. There are already several available optical contrast agents (such as fluorochromes and nano particles) and chromogenic disease biomarkers that can be readily adopted for use in multispectral photoacoustic tomography (MSOT) ${ }^{36}$. Our current novel 4-D PAT system uses custom-made high frequency transducer arrays with a spherical configuration covering an angle of $360^{\circ}$ in $\mathrm{x}-\mathrm{y}$ plane and of greater than $90^{\circ}$ in $\mathrm{y}-\mathrm{z}$ plane. It provides a higher spatial resolution and a 10 times bigger imaging volume relative to the previously reported PAT prototype ${ }^{28}$. In our system, there is no mechanical scanning needed along the axial $\mathrm{z}$ direction for $3 \mathrm{D}$ volumetric imaging. So the temporal resolution for $3 \mathrm{D}$ volumetric imaging is up to $3 \mathrm{~Hz}$ which is only limited by the current laser repetition rate. Thus, 4D PAT techniques could also help reduce the development time of new drugs and achieve faster clinical proofs-of-concept.

A key advantage of our noninvasive 4D PAT system for epileptiform event monitoring is the ability to image in real time. Seizures involve complex interactions of large groups of neurons and continually change throughout the event ${ }^{37}$. By achieving fast data acquisition, we will be able to observe transient alterations in metabolism and perfusion associated with interictal events. These events are not observable with current imaging systems such as fMRI, PET and SPECT. Our initial results indicate that there are generalized increases in photoacoustic signals associated with interictal events. Specifically, for the first time simultaneous EEG and PAT mapping of an epileptiform event in vivo were allowed in the experiments. We found that each interictal spike has a discrete photoacoustic correlate (Fig. 5b). The current imaging speed was limited by the $10 \mathrm{~Hz}$ laser pulse-repetition-rate. Since considerably faster lasers of $\sim 500 \mathrm{~Hz}$ pulse-repetition-rates are now commercially available, the temporal resolution of PAT will eventually reach a level of milliseconds. The 3-D, real-time imaging ability of our 4-D PAT shows the potential to study individual patients with spontaneously occurring epileptic discharges over distributed brain regions, and will likely help us to better understand the underlying mechanisms of the generation, propagation, and termination of epileptic discharges in three-dimension space.

With 4D PAT, real-time temperature monitoring of tumors treated with NIR-absorbing, non-bleaching nanoshells, can be used to ensure the successful thermal destruction of tumors. The 4D PAT method for volumetrically determining temperature distribution 
$\mathbf{a}$
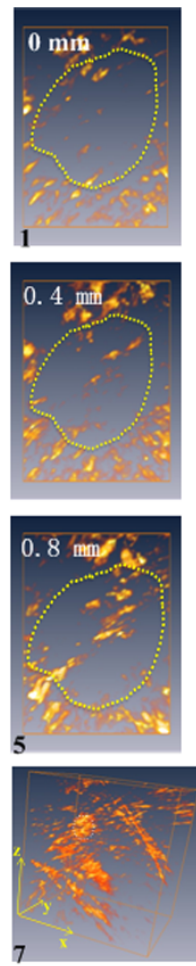
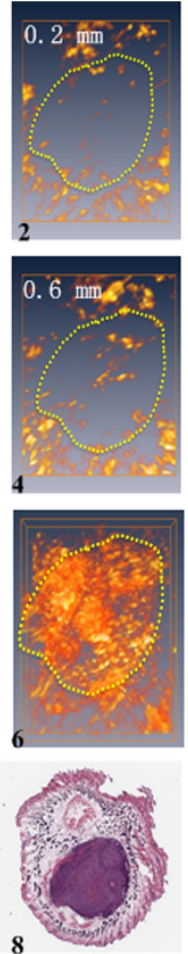

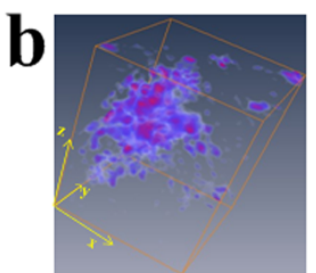

$90 \mathrm{~s}$

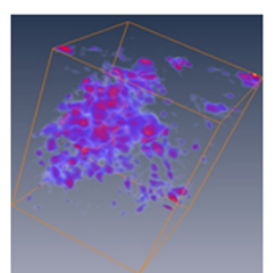

$120 \mathrm{~s}$

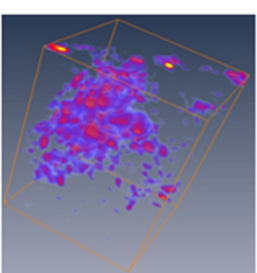

$150 \mathrm{~s}$

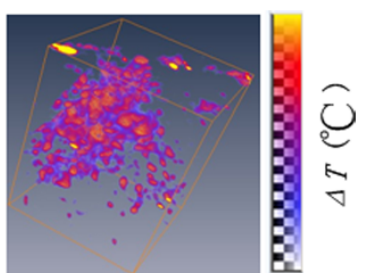

$180 \mathrm{~s}$

c

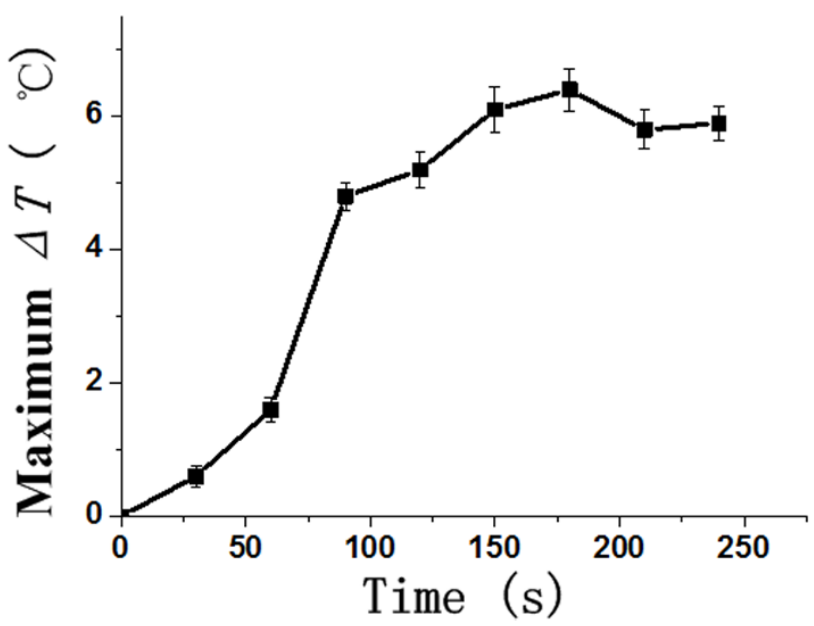

Figure 6 Tumor margin detection and thermal therapy monitoring. (a)Three-dimensional stack of consecutive photoacoustic image sections from a breast tumor on mouse, the $0.2-\mathrm{mm}$ slice corresponds to sections $1-5$. Maximum intensity projection of the entire $\mathrm{z}$ stack is shown in 6 ; 7 is the $3 \mathrm{D}$ reconstruction of the entire z-stack. Yellow dash circle represents the tumor margin in different slices as in 1-6 are correlated well with tumor histology in 8. (b) 3D photoacoustic-based thermal images at different time point of $90 \mathrm{~s}, 120 \mathrm{~s}, 150 \mathrm{~s}$ and $180 \mathrm{~s}$ after photothermal therapy, respectively.

(c) Photoacoustic measurement of temperature rise during photothermal therapy, results in maximum temperature rise of $\sim 6.6^{\circ} \mathrm{C}$ within the tumor, Error bars ( \pm s.d.) were calculated from 5 consecutive measurements.

during treatment would greatly enhance the ability to uniformly heat tumors at therapeutic levels. Histologic examination reveals that PAT estimation of tissue damage agrees well with experimental findings, demonstrating its potential utility in determining tissue damage during therapy. This will allow therapy regimens to be tailored to ensure complete thermal destruction of tumors. However, it is necessary to point out that the influence of depth dependent light attenuation, change of sound-speed and tissue absorption due to the thermal therapy should be evaluated in future studies with our quantitative photoacoustic image reconstruction method ${ }^{38,39}$. The radial resolution of approximately $190 \mu \mathrm{m}$ is only bandwidth limited and is thus spatially invariant, in good agreement with the theoretically predicted value ${ }^{40}$. The transverse resolution however varies significantly from $\sim 270 \mu \mathrm{m}$ in the center of the array, to approximately $400 \mu \mathrm{m}$ at a distance of $10 \mathrm{~mm}$ from the center. The reason for this is the reduced elevational aperture $\left(90^{\circ}\right)$ of the system, which provides relatively limited view in this direction. A larger elevation angle and more transducer elements could therefore improve the resolution. It is easy to further improve the spatial resolution by simply increasing the number of transducers in the current transducer array interface in our next generation imaging system.

This work represents a major technological and scientific advance in photoacoustic imaging. Previous attempts to perform real time 3D tracking of dynamic physiology and pathology phenomena in living tissue in 3-D were not successful due to temporal resolution and/or imaging depth limitations. The high spatial and temporal sampling of this novel 4D-PAT system allows for complete mapping of hemodynamic and thermodynamic events in vivo. The noninvasiveness and depth capabilities of this $4 \mathrm{D}-\mathrm{PAT}$ system allows monitoring of drug delivery and cancer therapy. 4D-PAT provides high-resolution imaging capabilities in real time in live tissue.

\section{Methods}

Animals. Male Sprague-Dawley rats (Harlan Labs, Indianapolis, IN) weighing 50-60 g on arrival were allowed one week to acclimate to the 12-h light/dark cycle and given food and water ad libitum. Before imaging, and while under anesthesia, the animal's head hair was removed using a hair removing lotion. The scalp and skull were kept intact. After PAT data acquisition, animals were sacrificed using pentobarbital (120 mg/kg, administered intraperitoneally(i.p.)). Subsequently, open-skull anatomical photography was performed. All procedures were approved by the University of Florida Animal Care and Use Committee and conducted in accordance with the National Institutes of Health Guide for the Care and Use of Experimental Animals.

Seizure induction and electrode implantation surgery. Generalized seizures were induced in rats $(\mathrm{n}=10)$ using intraperitoneal injection of pentylenetetrazol (PTZ). Cortical local field potentials were recorded 5 min before each injection and continued for up to $30 \mathrm{~min}$ post-injection. Rats were anesthetized by intraperitoneal injection of urethane $(1 \mathrm{~g} / \mathrm{kg}$ of body weight). Two $300 \mu \mathrm{m}$ diameter stainless steel screw electrodes were implanted in each rat skull to obtain potential data subdural multichannel cortical local field. Electrodes were placed at $-0.3 \mathrm{~mm}$ posterior; $3 \mathrm{~mm}$ lateral (right) of bregma and $1 \mathrm{~mm}$ ventral, based on coordinates from a rat brain atlas $^{40}$. In addition, one $300 \mu \mathrm{m}$ diameter stainless steel electrode (FHC, Bowdoin, $\mathrm{ME})$ was implanted as a reference into the midline occipital bone. Cortical local field potentials were obtained using a Tucker Davis Pentusa (Tucker Davis Technologies, Alachua, FL) neural recording system at $12 \mathrm{kHz}$, digitized with 16 bits of resolution, and band pass filtered from 0.5 to $6 \mathrm{kHz}$.

Drug delivery monitoring. Indocyanine green (ICG), or cardiogreen, is a U.S. Food and Drug Administration (FDA)-approved, nontoxic, organic dye used for medical diagnostics. The absorption spectrum of ICG bound with plasma proteins is $805 \mathrm{~nm}$. ICG in phosphate buffered saline $(\mathrm{pH}=7.4)$ was injected intravenously at a dosage of $0.25 \mathrm{ml} / 100 \mathrm{~g}$ body weight, which produced an estimated ICG blood concentration of $1 \times 10^{-5} \mathrm{M}$. After injecting ICG, a series of PA images was obtained to dynamically monitor ICG uptake in the rat brain.

Thermal therapy and temperature monitoring. For thermal therapy studies, one-month old BALB/C mice were injected in the right flank with EMTCL2 human cancer cells (about $5 \times 10^{6}$ cells) and tumors were allowed to grow for 15 days to a size of $10 \mathrm{~mm}$ in diameter. Sliver nanoplates were used for thermal therapy due to their 
enhanced thermal stability and enhanced photoacoustic signal response. By precisely controlling the plate diameter and thickness, the nanoplate's optical resonance can be tuned to peak at specific wavelengths at $785 \mathrm{~nm}$. The injected volume of nanoparticle solution was $200 \mu \mathrm{L}$, and the injected concentration was $0.08 \mathrm{mg} / \mathrm{ml}$. The nanoparticles were intradermally injected at the tumor site. A CW laser $(785 \mathrm{~nm}$, $80 \mathrm{~mW}$, Model DL7140-201S, Sanyo, Japan) was used for photothermal therapy. The 4-D photoacoustic imaging setup for thermal therapy and temperature monitoring in vivo on mouse tumor model is shown in Supplemental Figure 2.

The thermal expansion of an absorbing medium heated by a short laser pulse with an incident laser fluence, $F_{0}$, induces a pressure rise, $P(z)$, in the irradiated volume upon stress confined irradiation conditions ${ }^{41}$,

$$
P(z)=\left(\frac{\beta c_{s}^{2}}{C_{p}}\right) \mu_{a} F(z)=\Gamma \mu_{a} F(z)=(A+B T(z)) k \mu_{a} F_{0} e^{\left(-\mu_{\text {off }} z\right)}
$$

where $\beta\left[1^{\circ} \mathrm{C}^{-1}\right]$ is the thermal expansion coefficient, $c_{s}$ the speed of sound, $C_{p}\left[\mathrm{~J} \mathrm{~g}^{-1}{ }^{\circ} \mathrm{C}^{-1}\right]$ the heat capacity at constant pressure, $F(z)\left[\mathrm{J} \mathrm{cm}^{-2}\right]$ the laser fluence and $\mu_{a}\left[\mathrm{~cm}^{-1}\right]$ the absorption coefficient of the medium. The expression $\beta c_{s}^{2} / C_{p}$ in equation (2) represents the Gruneisen parameter, $\Gamma$, with an almost linear dependence on temperature. $A$ and $B$ are constants and $T(z)$ is the temperature distribution in tissue. $k$ is the parameter resulting from multiple scattering in tissue and is dependent on the absorption and scattering coefficients. The factor $e^{\left(-\mu_{\text {eff }} z\right)}$ represents exponential attenuation of the optical radiation in the medium. Equation (2) is valid under the condition of stress-confinement when pressure relaxation is negligible during the heat deposition.

Prior to taking experimental measurements, tissue temperatures were measured to calibrate the relationship between temperature and photoacoustic signal amplitude. During experiments, tissue samples were immersed in a water tank maintained at $37^{\circ} \mathrm{C}$ and thermistors were inserted into the centers of tissue samples to measure temperature. Baseline ultrasound and photoacoustic frames were acquired before photoacoustic frames were recorded at each temperature (see supplemental data Figure 2, online).

Image analysis. A custom software utility was written and incorporated into the computer software package MATLAB (MathWorks, Massachusetts, USA) to analyze and display recorded data. Amira (version 5.3.3, TGS Template Graphics Software) was used for 3D reconstruction of the PA slices. Movies were formed by merging time serials of 3D PAT images from Amira using custom Matlab code. The image color scale was determined by the photoacoustic signal intensity in arbitrary units.

1. Wang, L. V. \& Hu, S. Photoacoustic tomography: in vivo imaging from organelles to organs. Science 335, 1458-1462 (2012).

2. Andreev, V. G., Karabutov, A. A. \& Oraevsky, A. A. Detection of ultrawide-band ultrasound pulses in optoacoustic tomography. IEEE Trans Ultrason Ferroelectr Freq Control 50, 1383-1390 (2003).

3. Bell, A. G. On the Production and Reproduction of Sound by Light. Am J Sci $\mathbf{2 0}$ (1880).

4. Beard, P. Biomedical photoacoustic imaging. Interface Focus 1, 602-631 (2011).

5. Xiang, L. et al. Real-time optoacoustic monitoring of vascular damage during photodynamic therapy treatment of tumor. J Biomed Opt 12, 014001 (2007).

6. Wang, B. et al. Plasmonic intravascular photoacoustic imaging for detection of macrophages in atherosclerotic plaques. Nano Lett 9, 2212-2217 (2009).

7. Yuan, Y., Yang, S. H. \& Xing, D. Optical-resolution photoacoustic microscopy based on two-dimensional scanning galvanometer. Applied Physics Letters 100, 023702 (2012).

8. Zhang, H. F., Maslov, K., Stoica, G. \& Wang, L. V. Functional photoacoustic microscopy for high-resolution and noninvasive in vivo imaging. Nat Biotechnol 24, 848-851 (2006).

9. Manohar, S. et al. Initial results of in vivo non-invasive cancer imaging in the human breast using near-infrared photoacoustics. Opt Express 15, 12277-12285 (2007).

10. Sun, Y., Sobel, E. S. \& Jiang, H. B. First assessment of three-dimensional quantitative photoacoustic tomography for in vivo detection of osteoarthritis in the finger joints. Medical Physics 38, 4009-4017 (2011).

11. Wang, X., Chamberland, D. L. \& Jamadar, D. A. Noninvasive photoacoustic tomography of human peripheral joints toward diagnosis of inflammatory arthritis. Opt Lett 32, 3002-3004 (2007).

12. Brecht, H. P. et al. Whole-body three-dimensional optoacoustic tomography system for small animals. J Biomed Opt 14, 064007 (2009).

13. Razansky, D., Buehler, A. \& Ntziachristos, V. Volumetric real-time multispectral optoacoustic tomography of biomarkers. Nat Protoc 6, 1121-1129 (2011).

14. Wang, X. et al. Noninvasive laser-induced photoacoustic tomography for structural and functional in vivo imaging of the brain. Nat Biotechnol 21, 803-806 (2003).

15. Buehler, A., Herzog, E., Razansky, D. \& Ntziachristos, V. Video rate optoacoustic tomography of mouse kidney perfusion. Opt Lett 35, 2475-2477 (2010).

16. Li, C. et al. Real-time photoacoustic tomography of cortical hemodynamics in small animals. J Biomed Opt 15, 010509 (2010).

17. Charboneau, J. W., Reading, C. C. \& Welch, T. J. CT and sonographically guided needle biopsy: current techniques and new innovations. AJR Am J Roentgenol 154, 1-10 (1990).
18. Su, J., Karpiouk, A., Wang, B. \& Emelianov, S. Photoacoustic imaging of clinical metal needles in tissue. J Biomed Opt 15, 021309 (2010).

19. Kim, C. et al. Handheld array-based photoacoustic probe for guiding needle biopsy of sentinel lymph nodes. J Biomed Opt 15, 046010 (2010).

20. Aboofazeli, M., Abolmaesumi, P., Mousavi, P. \& Fichtinger, G. A New Scheme for Curved Needle Segmentation in Three-Dimensional Ultrasound Images. Proc IEEE Int Symp Biomed Imaging 2009, 1067-1070 (2009).

21. Shen, F. et al. Three-dimensional sonography with needle tracking: role in diagnosis and treatment of prostate cancer. JUltrasound Med 27, 895-905 (2008).

22. Zhang, Q. et al. Non-invasive imaging of epileptic seizures in vivo using photoacoustic tomography. Phys Med Biol 53, 1921-1931 (2008).

23. Joshi, P. P. et al. Molecular therapeutic agents for noninvasive photoacoustic image-guided photothermal therapy. Conf Proc IEEE Eng Med Biol Soc 2009, 4106-4109 (2009).

24. Lu, W. et al. Effects of photoacoustic imaging and photothermal ablation therapy mediated by targeted hollow gold nanospheres in an orthotopic mouse xenograft model of glioma. Cancer Res 71, 6116-6121 (2011).

25. Shah, J. et al. Photoacoustic imaging and temperature measurement for photothermal cancer therapy. J Biomed Opt 13, 034024 (2008).

26. Lou, C. \& Xing, D. Temperature monitoring utilising thermoacoustic signals during pulsed microwave thermotherapy: a feasibility study. Int J Hyperthermia 26, 338-346 (2010).

27. Wang, Q., Tolkach, A. \& Kulozik, U. Quantitative assessment of thermal denaturation of bovine alpha-lactalbumin via low-intensity ultrasound, HPLC, and DSC. J Agric Food Chem 54, 6501-6506 (2006).

28. Buehler, A., Dean-Ben, X. L., Claussen, J., Ntziachristos, V. \& Razansky, D. Three-dimensional optoacoustic tomography at video rate. Optics Express 20 , 22712-22719 (2012).

29. Ephrat, P., Roumeliotis, M., Prato, F. S. \& Carson, J. J. Four-dimensional photoacoustic imaging of moving targets. Opt Express 16, 21570-21581 (2008).

30. Wang, B., Xiang, L., Jiang, M. S., Zhang, Q., Carney, P. R. \& Jiang, H. Photoacoustic tomography system for noninvasive real-time three-dimensional imaging of epilepsy. Biomed Opt EXPRESS 3, 1427 (2012).

31. Zhou, Q. Z., Ji, X. R. \& Xing, D. Full-field 3D photoacoustic imaging based on plane transducer array and spatial phase-controlled algorithm. Medical Physics 38, 1561-1566 (2011)

32. Jiang, $\mathrm{H}$. \& Xu, Y. Phase-contrast imaging of tissue using near-infrared diffusing light. Medical Physics 30, 1048-1051 (2003).

33. Xi, L. et al. Evaluation of breast tumor margins in vivo with intraoperative photoacoustic imaging. Opt Express 20, 8726-8731 (2012).

34. Su, J., Karpiouk, A., Wang, B. \& Emelianov, S. Photoacoustic imaging of clinical metal needles in tissue. Journal of Biomedical Optics 15, 021309 (2010).

35. Kim, C. et al. Handheld array-based photoacoustic probe for guiding needle biopsy of sentinel lymph nodes. Journal of Biomedical Optics 15, 046010 (2010).

36. Razansky, D., Vinegoni, C. \& Ntziachristos, V. Multispectral photoacoustic imaging of fluorochromes in small animals. Opt Lett 32, 2891-2893 (2007).

37. Schwartz, T. H. \& Bonhoeffer, T. In vivo optical mapping of epileptic foci and surround inhibition in ferret cerebral cortex. Nat Med 7, 1063-1067 (2001).

38. Yuan, Z., Wang, Q. \& Jiang, H. B. Reconstruction of optical absorption coefficient maps of heterogeneous media by photoacoustic tomography coupled with diffusion equation based regularized Newton Method. Optics Express 15, 18076-18081 (2007).

39. Yao, L., Sun, Y. \& Jiang, H. B. Quantitative photoacoustic tomography based on the radiative transfer equation. Optics Letters 34, 1765-1767 (2009).

40. Xu, M. \& Wang, L. V. "Analytic explanation of spatial resolution related to bandwidth and detector aperture size in thermoacoustic or photoacoustic reconstruction," Phys. Rev. E Stat. Nonlin. Soft Matter Phys. 67(5), 056605 (2003).

41. Larina, I. V., Larin, K. V. \& Esenaliev, R. O. Real-time optoacoustic monitoring of temperature in tissues. Journal of Physics D-Applied Physics 38, 2633-2639 (2005).

\section{Acknowledgements}

This research was supported in part by the J. Crayton Pruitt Family endowment fund. The authors thank Colin Carpenter and Susan Dunlap for helpful discussions.

\section{Author contributions}

Liangzhong Xiang and Huabei Jiang conceived the study, Liangzhong Xiang and Bo Wang performed field work for imaging system development, Liangzhong Xiang and Lijun Ji performed animal experiments, Liangzhong Xiang performed statistical analysis, made figures for the paper, and Liangzhong Xiang and Huabei Jiang directed the study and wrote the paper.

\section{Additional information}

Supplementary information accompanies this paper at http://www.nature.com/ scientificreports

Competing financial interests: The authors declare no competing financial interests.

License: This work is licensed under a Creative Commons 
Attribution-NonCommercial-NoDerivs 3.0 Unported License. To view a copy of this license, visit http://creativecommons.org/licenses/by-nc-nd/3.0/
How to cite this article: Xiang, L., Wang, B., Ji, L. \& Jiang, H. 4-D Photoacoustic Tomography. Sci. Rep. 3, 1113; DOI:10.1038/srep01113 (2013). 